\title{
Relationship of porcine IGF2 imprinting status to DNA methylation at the H19 DMD and the IGF2 DMRs 1 and 2
}

\author{
Martin H Braunschweig ${ }^{*}$, Marta Owczarek-Lipska and Nasikhat Stahlberger-Saitbekova
}

\begin{abstract}
Background: Porcine IGF2 and the H19 genes are imprinted. The IGF2 is paternally expressed, while the H19 gene is maternally expressed. Extensive studies in mice established a boundary model indicating that the $\mathrm{H} 19$ differentially methylated domain (DMD) controls, upon binding with the CTCF protein, reciprocal imprinting of the IGF2 and the H19 genes. IGF2 transcription is tissue and development specific involving the use of 4 promoters. In the liver of adult Large White boars IGF2 is expressed from both parental alleles, whereas in skeletal muscle and kidney tissues we observed variable relaxation of IGF2 imprinting. We hypothesized that IGF2 expression from both paternal alleles and relaxation of IGF2 imprinting is reflected in differences in DNA methylation patterns at the H19 DMD and IGF2 differentially methylated regions 1 and 2 (DMR1 and DMR2).
\end{abstract}

Results: Bisulfite sequencing analysis did not show any differences in DNA methylation at the three porcine CTCF binding sites in the H19 DMD between liver, muscle and kidney tissues of adult pigs. A DNA methylation analysis using methyl-sensitive restriction endonuclease Sacll and 'hot-stop' PCR gave consistent results with those from the bisulfite sequencing analysis. We found that porcine H19 DMD is distinctly differentially methylated, at least for the region formally confirmed by two SNPs, in liver, skeletal muscle and kidney of foetal, newborn and adult pigs, independent of the combined imprinting status of all IGF2 expressed transcripts. DNA methylation at CpG sites in DMR1 of foetal liver was significantly lower than in the adult liver due to the presence of hypomethylated molecules. An allele specific analysis was performed for IGF2 DMR2 using a SNP in the IGF2 3'-UTR. The maternal IGF2 DMR2 of foetal and newborn liver revealed a higher DNA methylation content compared to the respective paternal allele.

Conclusions: Our results indicate that the IGF2 imprinting status is transcript-specific. Biallelic IGF2 expression in adult porcine liver and relaxation of IGF2 imprinting in porcine muscle were a common feature. These results were consistent with the IGF2 promoter P1 usage in adult liver and IGF2 promoter P2, P3 and P4 usages in muscle. The results showed further that bialellic IGF2 expression in liver and relaxation of imprinting in muscle and kidney were not associated with DNA methylation variation at and around at least one CTCF binding site in H19 DMD. The imprinting status in adult liver, muscle and kidney tissues were also not reflected in the methylation patterns of IGF2 DMRs 1 and 2.

\section{Background}

Porcine insulin-like growth factor 2 (IGF2) and $H 19$ genes are reciprocally imprinted in most tissues. In mice, these two genes share common endodermal and mesodermal enhancers and the mouse Igf2 gene is also paternally expressed in most tissues whereas the $H 19$

\footnotetext{
* Correspondence: martin.braunschweig@vetsuisse.unibe.ch Institute of Genetics, Vetsuisse Faculty, University of Bern, 3001 Berne, Switzerland
}

gene is maternally transcribed [1-3]. Mice lacking the Igf2 gene weighed about $40 \%$ less than their litter mates [4]. The $H 19$ gene expresses a non-protein-coding RNA and is located $88.1 \mathrm{~kb}$ downstream of IGF2 [5,6]. Recently, it was found that $\mathrm{H} 19$ transcripts can function as microRNA precursors [7].

The pig INS-IGF2-H19 imprinting cluster is highly homologous to the corresponding human gene cluster and is thus a good model to study epigenetic 
mechanisms [5]. Recently, a quantitative trait nucleotide (QTN) at position IGF2-intron3-3072 was identified and various antisense transcripts originate from the paternal allele demonstrating the complex transcription from this gene $[8,9]$.

An extensive number of studies have been conducted to elucidate the epigenetic mechanisms of IGF2 and H19 which are thought to be co-ordinately regulated, both in terms of their expression patterns and their reciprocal imprinting (for review see [3]). It was shown by deletions in mice that a region of paternal-specific DNA methylation (differentially methylated domain, DMD) upstream of $H 19$ is an epigenetic mark required for imprinting of $I G F 2$ and $H 19$ [10,11]. Bell and Felsenfeld [12] reported that activity of H19 DMD depends upon the vertebrate eleven-zinc finger protein CTCF that binds to this DMD and mediates the function of the boundary/insulator element. They also found that methylated CpG sites at the CTCF binding site abolished binding in vitro. Based on this finding Bell and Felsenfeld [12] developed a model explaining the reciprocal imprinting. On the maternal allele the enhancer downstream of $H 19$ has no access to the IGF2 promoters due to the boundary function of CTCF proteins bound to the unmethylated DMD whereas the H19 gene can still be transcribed. On the paternal allele DNA methylation at the H19 DMD eradicates the boundary function which leads to IGF2 gene transcription and silencing of the $\mathrm{H} 19$ gene. These findings were made simultaneously using transgenic mice and cell culture and contributed to establish the boundary model [13]. More recently it was demonstrated that differentially methylated regions in the mouse $I g f 2$ and $H 19$ genes interact in an epigenetically regulated manner that partition maternal and paternal alleles into distinct loops. The maternal allele H19 DMD interacts with Igf2 DMR1 allowing maternal $H 19$ to be expressed while the paternal H19 DMD interacts with Igf2 DMR2, allowing Igf2 to be expressed and leaves the $H 19$ gene silent. This model was named the chromatin loop model [14].

A DMD between -1.1 and $-2.6 \mathrm{~kb}$ upstream of the H19 transcription start site has also been found to regulate porcine reciprocal transcription of $H 19$ and IGF2 via a CTCF protein that binds to the unmethylated maternal allele (Figure 1). In the pig H19 DMD, three CTCF-binding motifs in P1, P2 and P3 repeats were identified by sequence homology to the human H19 DMD [5]. Relaxation of IGF2 imprinting was observed in skeletal muscle of 4 month old pigs compared to nearly complete monoallelic expression in prenatal skeletal muscle [8]. Wrzeska et al. [15] reported biallelic IGF2 expression in adult porcine liver and brain and monoallelic IGF2 expression in muscle and kidney tissues of adult pigs. Recently, it was reported that IGF2 transcripts from promoter P1 are also biallelically expressed in tissues of week-old pigs whereas in most other tissues, including skeletal muscle and kidney, IGF2 was monoallelically expressed [16]. Biallelic expression of $I g f 2 / I G F 2$ has been reported for choroids plexus and leptomeninges of the mouse as well as in postnatal and normal adult human liver $[1,17,18]$. A recent report by Wu et al. [19] confirmed biallelic IGF2 expression in normal human liver and showed a medium-methylated H19 DMD profile with a hypermethylated paternal and a hypomethylated maternal allele.

In an ongoing study we investigated the combined imprinting status of all IGF2 expressed transcripts in liver, skeletal muscle and kidney tissues of adult boars. We hypothesized that an alteration in IGF2 imprinting status might be reflected in DNA methylation variations at differentially methylated regions as suggested by the boundary and chromatin loop models. To test this hypothesis we studied the association between the IGF2 imprinting status in three different tissue samples of six boars and their DNA methylation of H19 DMD, IGF2 DMR1 and DMR2 (Figure 1). Furthermore, we included samples from two foetus and two newborn pigs in order to examine IGF2 imprinting and DNA methylation at these differentially methylated regions during development. We were curious to see whether the imprinting status of IGF2 in liver, skeletal muscle and kidney at different developmental stages is also reflected in the DNA methylation patterns of H19 DMD and in particular at CTCF binding sites as well as in IGF2 DMR1 and IGF2 DMR2.

\section{Results}

\section{Imprinting status of IGF2}

We used the SWC9 microsatellite marker with alleles $236 \mathrm{bp}$ and $247 \mathrm{bp}$ to investigate the combined imprinting status of IGF2 gene expression in liver, skeletal muscle and kidney (Figure 1). The SWC9 microsatellite is located in the 3' UTR of the IGF2 gene and its sequence is common to all IGF2 transcripts originating from different promoter usages in a specific tissue and developmental stages. We investigated two pig foetuses, two newborn piglets and six boars that were heterozygous for the $S W C 9$ marker. All but one boar received the $S W C 9$ allele 247 bp from their fathers and the SWC9 $236 \mathrm{bp}$ allele from their mothers. We established a standard curve with a dilution series of DNA from alternative homozygous individuals for the SWC9 marker [20]. As a measure for the imprinting status we calculated the $\log 2$ ratio of the $S W C 9236$ bp peak area to the SWC9 247 bp peak area and calculated the corresponding ratio of SWC9 236 bp allele to SWC9 247 bp based on the standard curve (Figure 2B). In Table 1 the ratios of allelic IGF2 expression for the different tissues and 


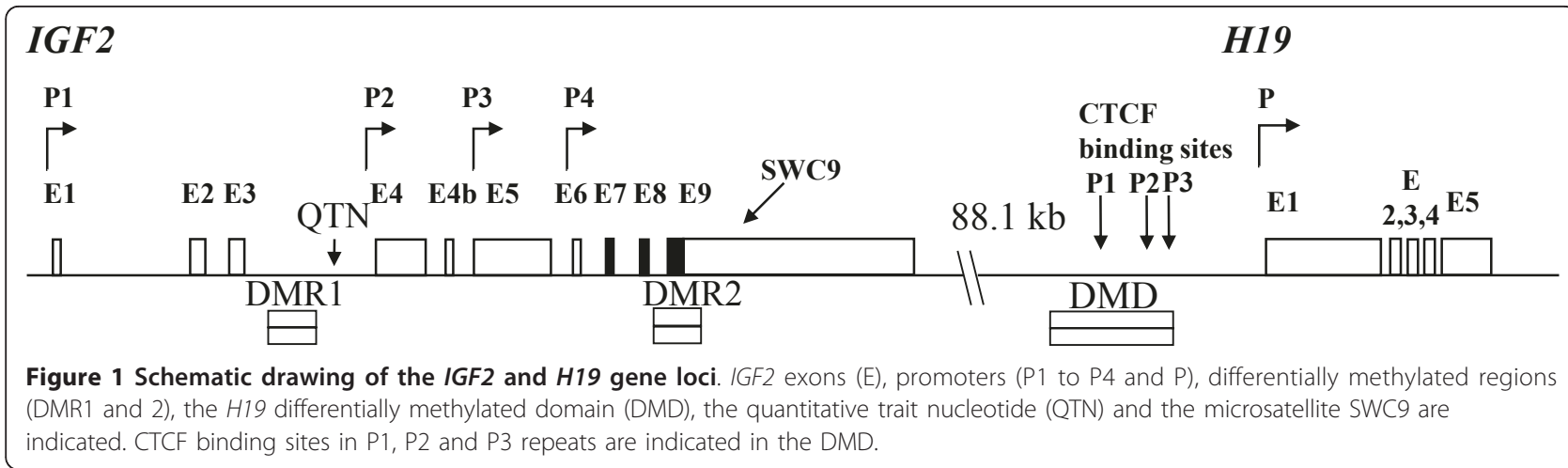

development stages are shown. This experiment showed that the imprinting status of IGF2 changes from paternal expression in the foetal liver to IGF2 expression from both parental alleles in the adult liver. Paternal IGF2 gene expression is mostly maintained in kidney tissue during development. In muscle tissue a trend of relaxation of imprinting during development is indicated. The considerable variation of these ratios is mostly explained by the small sample size and a few samples that substantially deviate from the mean but were nonetheless kept in the analysis. IGF2 imprinting in liver, muscle and kidney of foetal, newborn and adult pigs is visualized by the $S W C 9$ microsatellite profiles in Figure 2A.

The observed biallelic IGF2 expression in these adult boars' liver is in agreement with previous reports showing biallelic expression of IGF2 in the livers of humans and pigs [15-19]. RT-PCR analysis of adult liver cDNA indicated that all 4 IGF2 promoters were used whereas cDNA from muscle and kidney revealed very low level of products after 40 PCR cycles for transcripts from promoter P1 and high level of products for transcripts from promoter P2, P3, and P4 (data not shown). This result is in agreement with previous findings from Amarger et al. and Li et al. $[5,16]$, however, from previous Northern blot analysis it is known that promoter $\mathrm{P} 1$ is predominantly used in adult pig liver and transcripts from promoter P2, P3 and P4 were not detected. The RT-PCR analysis of microsatellite $S W C 9$ is a semiquantitative approach to investigate IGF2 imprinting status and the results conclusively indicate that IGF2 imprinting is reversed similarly to biallelic expression in adult liver and it is relaxed to different degrees in muscle and kidney tissues during development.

\section{Bisulfite sequencing analysis of $H 19$ DMD}

Bisulfite sequencing of porcine H19 DMD indicated that the region was indeed differentially methylated from about $-1 \mathrm{~kb}$ to $-3 \mathrm{~kb}$ from the start site of $H 19$ exon 1 (AY044827.1). We sequenced bisulfite treated DNA from four overlapping PCR fragments derived from adult liver, skeletal muscle and kidney DNA and found distinct hyper- and hypomethylated molecules in each of the four fragments. This strongly suggests that H19 DMD extended at least over this region (PCR primers H19_DMD_1 to H19_DMD_4, Additional file 1 Table S1). In the two PCR fragments spanned by PCR primer pairs (H19_DMD_2 and H19_DMD_3) two SNPs (AY044827.1:g.32530C $>$ T and AY044827.1:g.32619G >A) were identified and thus the parental alleles deduced which confirmed the DMD. This result is in perfect agreement with the boundary model which explains the mechanism by which CTCF's binding at the H19 DMD mediates chromatin insulator function and thus maintains the reciprocal imprinting of the IGF2 and H19 genes. We have analysed DNA methylation and in particular CpG sites at the CTCF binding site in the P2 repeat in liver, muscle and kidney tissues of foetal, newborn and adult pigs [5]. In Figure 3 DNA methylation patterns of the bisulfite sequencing analysis are shown as a 'lollipop' graphic. There is no apparent difference in DNA methylation at these three CpG sites within the CTCF binding site P2 between liver, skeletal muscle and kidney tissues in all development stages. Furthermore, a bisulfite sequencing analysis from 7 to 13 clones from each of the three tissues liver, kidney and muscle of three adult boars indicated hyper- and hypomethylatd CTCF binding sites in $\mathrm{P} 1$ and $\mathrm{P} 3$ repeats. This result is consistent with a medium-methylated H19 DMD profile, although we did not find a DNA polymorphism to determine the parental origin of these clones (data not shown). Sanger sequencing of bisulfite treated DNA molecules is laborious and only a relatively small number of molecules can be sequenced. There may also be heteroduplex artifacts present during PCR amplification of bisulfite treated DNA molecules because the bacterial host's mismatch repair system can convert paternal and maternal alleles into single hybrid sequences during cloning. To account for these drawbacks we performed a DNA methylation analysis using SacII and nonradioactive 'hot-stop' PCR [21]. 


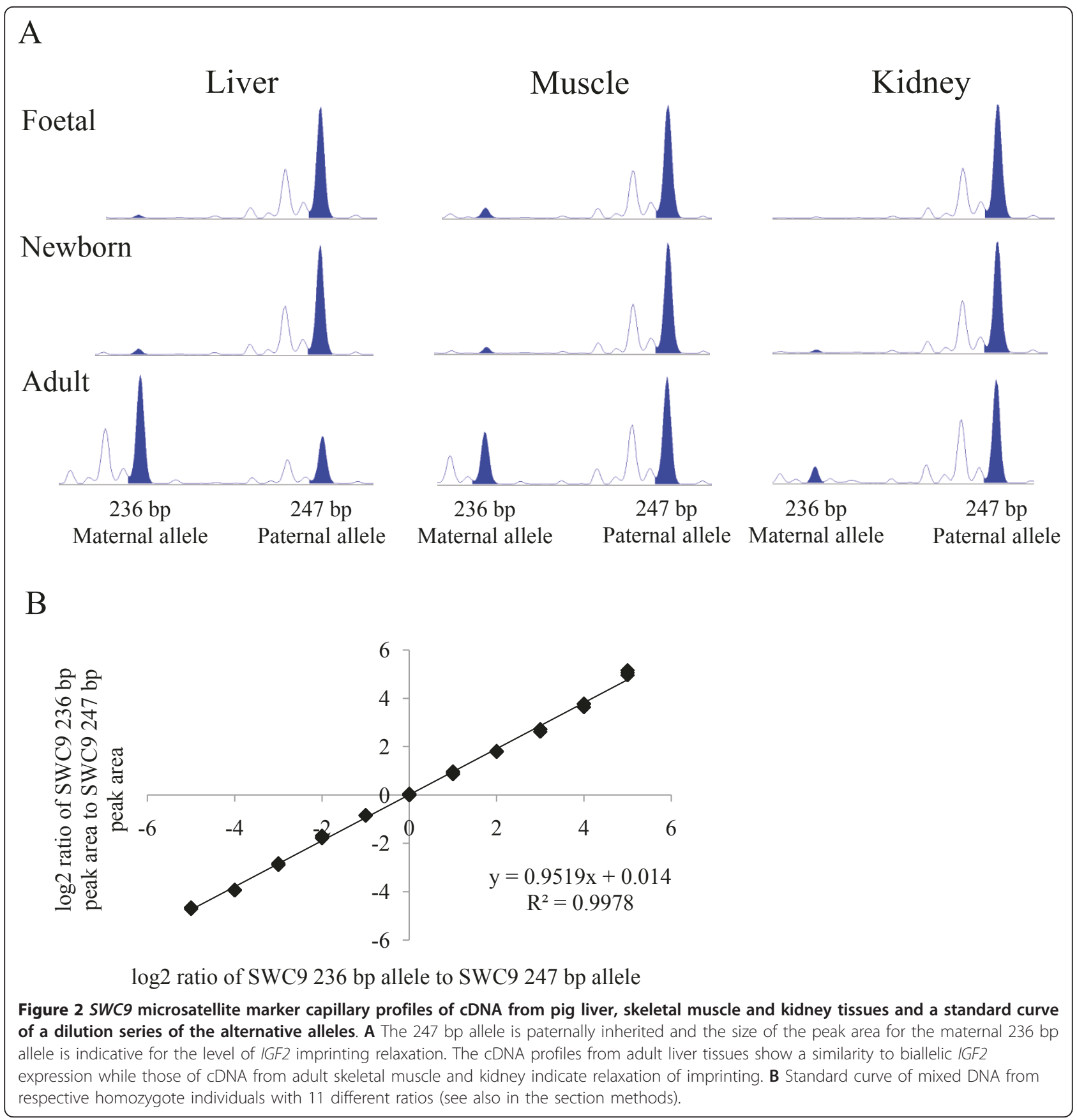

This assay discriminates between DNA methylation at maternal and paternal derived alleles at the CTCF binding site in the P2 repeat.

\section{DNA methylation analysis using methyl-sensitive restriction endonuclease}

In Figure 4 the assay of the DNA methylation analysis using a methyl-sensitive restriction endonuclease is shown. SacII recognizes the CTCF binding site in the P2 repeat and does not cleave if one or both of the two
CpG sites are methylated, i.e. the amplified products represent at least one methylated $\mathrm{CpG}$ at the binding site. Paternal and maternal alleles are discriminated by a restriction site for Bst $U \mathrm{II}$. This assay detects the ratio between paternal and maternal CpG methylation at the P2 repeat in H19 DMD. The proportions of unmethylated CpG sites in the CTCF binding site in the P2 repeat are not accounted for by this method. In lane 1 an undigested $464 \mathrm{bp} \mathrm{PCR}$ product is shown that is 29 bp longer than the digested longer allele since there is a 
Table 1 Imprinting status in different pig tissues and for three development stages expressed as ratio of paternal to maternal IGF2 gene expression

\begin{tabular}{ll}
\hline Tissue & $\begin{array}{l}\text { Ratio paternal to maternal gene expression } \\
( \pm \text { SD) }\end{array}$ \\
\hline Adult liver $(\mathrm{N}=6)$ & $4.2( \pm 6.2)$ \\
Newborn liver $(\mathrm{N}=2)$ & $21.3( \pm 0.01)$ \\
Foetal liver $(\mathrm{N}=2)$ & Paternal expression \\
Adult muscle $(\mathrm{N}=6)$ & $9.2( \pm 6.2)$ \\
Newborn muscle $(\mathrm{N}$ & $19.9( \pm 6.0)$ \\
$=2)$ & \\
Foetal muscle $(\mathrm{N}=2)$ & Paternal expression $(\mathrm{N}=1)$ and $14(\mathrm{~N}=1))$ \\
Adult kidney $(\mathrm{N}=6)$ & $22.9( \pm 10.2)$ \\
Newborn kidney $(\mathrm{N}=$ & 34.0 \\
1) & \\
Foetal kidney $(\mathrm{N}=2)$ & Paternal expression \\
\hline
\end{tabular}

second BstUI restriction site located in the CTCF binding site. Lanes 2 and 3 show the 137 bp fragment from foetal tissues indicating paternal hypermethylation and maternal hypomethylation at the CTCF P2 binding site. Also in newborn pig tissues (lanes 4 and 5, fragments of $137 \mathrm{bp}$ ) and in the tissues of adult pigs (lanes 6 and 7, $137 \mathrm{bp}$ and $435 \mathrm{bp}$, the later having the alternative paternal allele methylated) paternal hypermethylation and maternal hypomethylation at this CTCF P2 binding sites is suggested. In lane 8 a control result of a 1:1 mix of DNA from respective homozygous CC and TT boars for the AY044827.1:g.32530.C>T polymorphism is shown. The results of this assay consistently indicate paternal hypermethylation and maternal hypomethylation at this CTCF binding site in the H19 DMD in the three tissues and for the three development stages.

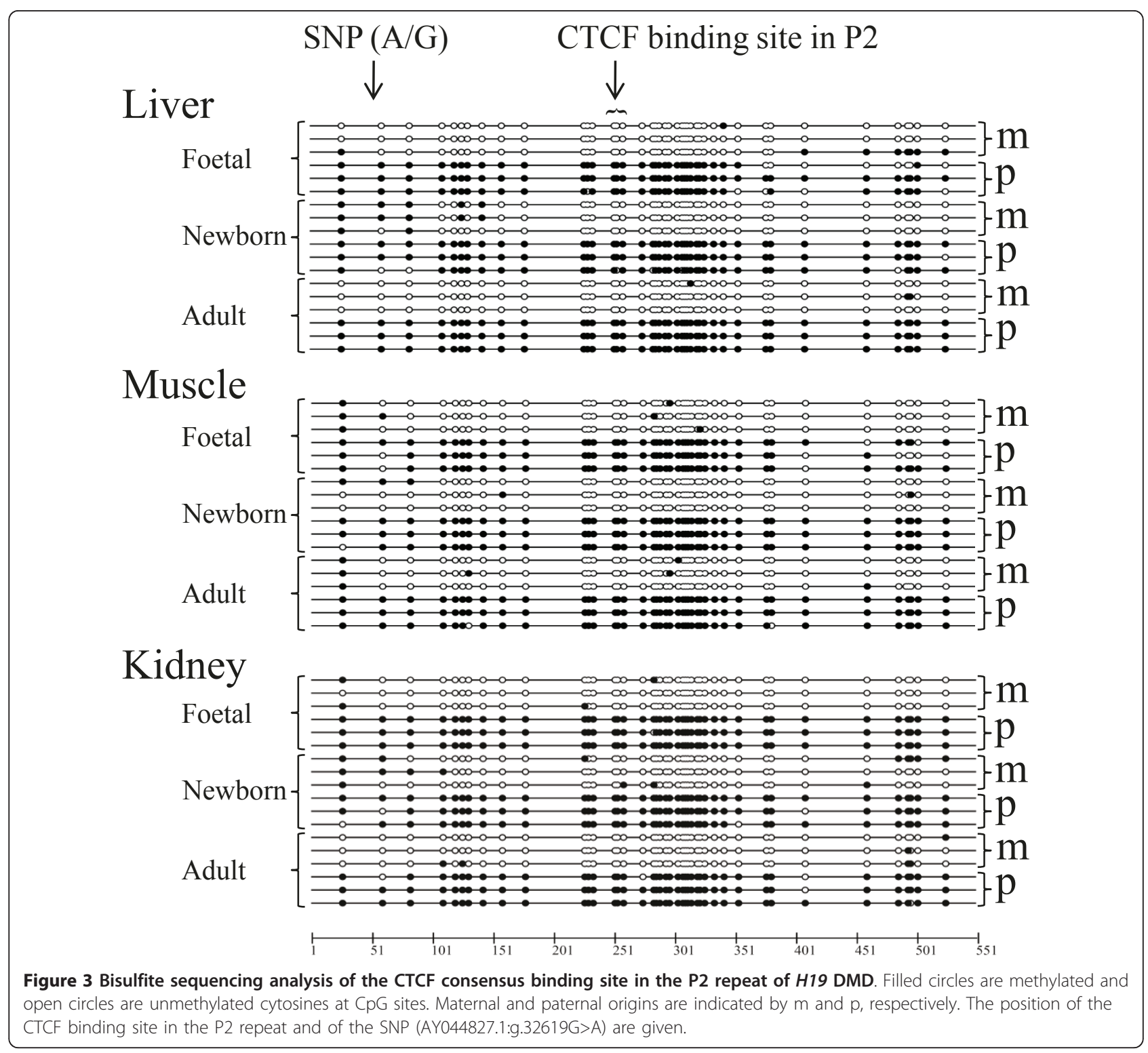




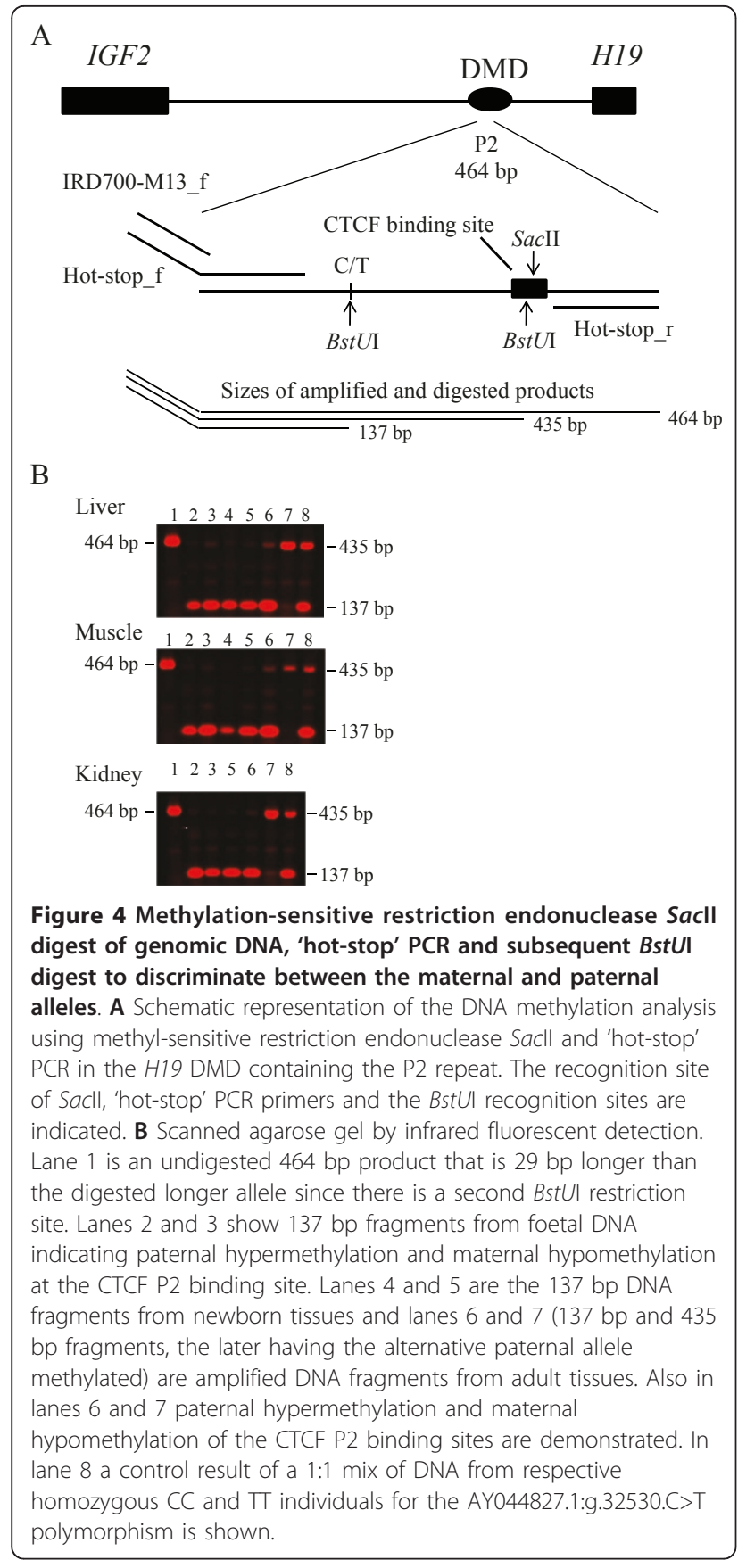

\section{Bisulfite sequencing analysis of IGF2 DMR1}

DNA polymorphisms that allowed the deduction of the parental origin of alleles in the IGF2 DMR1 were not found. Nevertheless we used bisulfite sequencing to analyse a considerable number of clones to search for specific DNA methylation patterns both between the three tissue samples and during development, which are supposed to be associated with the IGF2 imprinting status. We compared DNA from between 7 and 37 single clones per tissue and developmental stage and could not find any significant difference between DMR1 methylation in muscle and kidney tissues within foetal, newborn and adult individuals (Wilcoxon two-sample test, twosided).

There was a significant difference in DNA methylation at DMR1 CpG sites between 22 clones from the foetal liver compared to that of 33 clones from the adult pig liver $(P<0.01$, Wilcoxon two-sample test, two-sided). A suggestive difference in DNA methylation at DMR1 was found between 22 clones from foetal liver tissues and 13 clones from livers of two newborn piglets $(P=0.06)$. A closer inspection of the data showed a small fraction of hypomethylated DMR1 clones in the foetal liver. This result must be interpreted cautiously due to the small sample number. DMR1 was hypermethylated with over $70 \%$ DNA methylation in all tissue samples except for foetal liver and the foetal muscle, which had 66\% (22 clones) and 68\% (22 clones) DNA methylation, respectively. Representative lollipop diagrams are given in Figure 5 for all tissues and the three developmental stages.

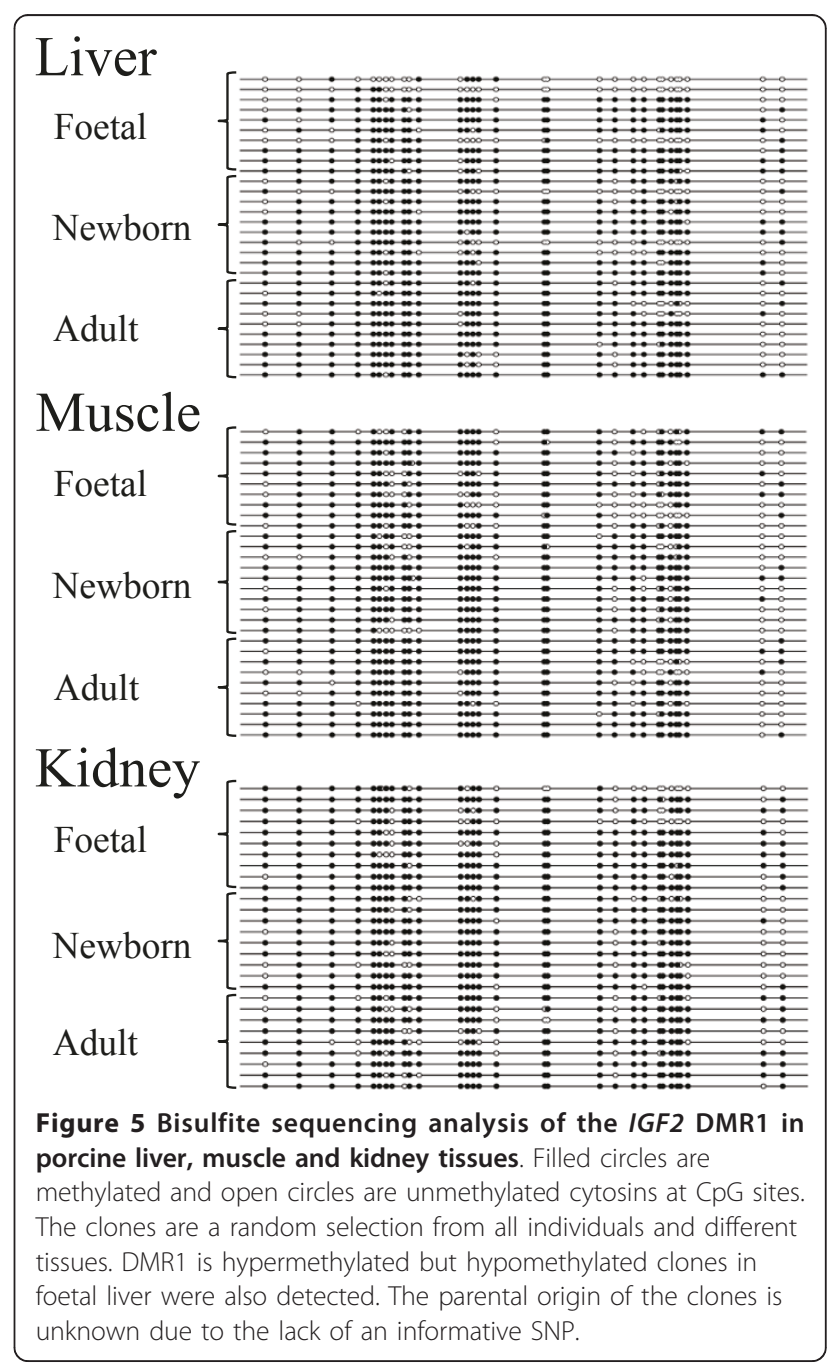


In summary, the DMR1 at the IGF2 locus was largely hypermethylated with some indication of hypomethylated DNA molecules in foetal liver.

\section{Bisulfite sequencing analysis of IGF2 DMR2}

We used the IGF2-exon9-612A>T SNP to determine the parental origin of DMR2 molecules from the bisulfite sequence analysis. In Figure 6 maternal and paternal profiles of the DNA methylation at each of the $17 \mathrm{CpG}$ sites are shown for three tissues and three developmental stages. Overall results showed hypermethylation of both parental DMR2 alleles. We focused on differences in DNA methylation of the two parental alleles in three tissues and during development. We found significantly higher DNA methylation along the $17 \mathrm{CpG}$ sites on the maternal allele in foetal (14 maternal clones versus 11 paternal clones) and newborn liver (24 maternal clones versus 23 paternal clones) compared to the paternal allele $(P<0.05$, Wilcoxon two-sample test, two-sided). However, it should be emphasised that both alleles were hypermethylated with $71 \%$ and $64 \%$ in foetal and $87 \%$ and $82 \%$ in the newborn liver for the respective maternal and the paternal alleles. There was a tendency that the maternal allele was also more methylated in foetal and newborn muscle and kidney tissues compared to the paternal allele. No significant differences in DNA methylation were observed between the parental alleles in the adult tissues.

\section{Discussion}

Van Laere et al. [8] and Wrzeska et al. [15] showed relaxation of imprinting in skeletal muscle tissues of 4 month old pigs and exclusive paternal IGF2 expression in the tissues of adult pigs' skeletal muscle, respectively. It is important to point out that insufficient PCR cycles, or template, may lead to the product arising from the maternal allele to go undetected, especially in the muscle and kidney, where relaxation of imprinting seems to increase with aging (Figure 2). This might be a reason for the conflicting data found by Van Laere et al. [8] and Wrzeska et al. [15]. In a comprehensive study the imprinting status of IGF2 and H19 were determined in 13 tissue samples of week-old piglets [16]. Li et al. (2008) [16] found biallelic IGF2 expression from

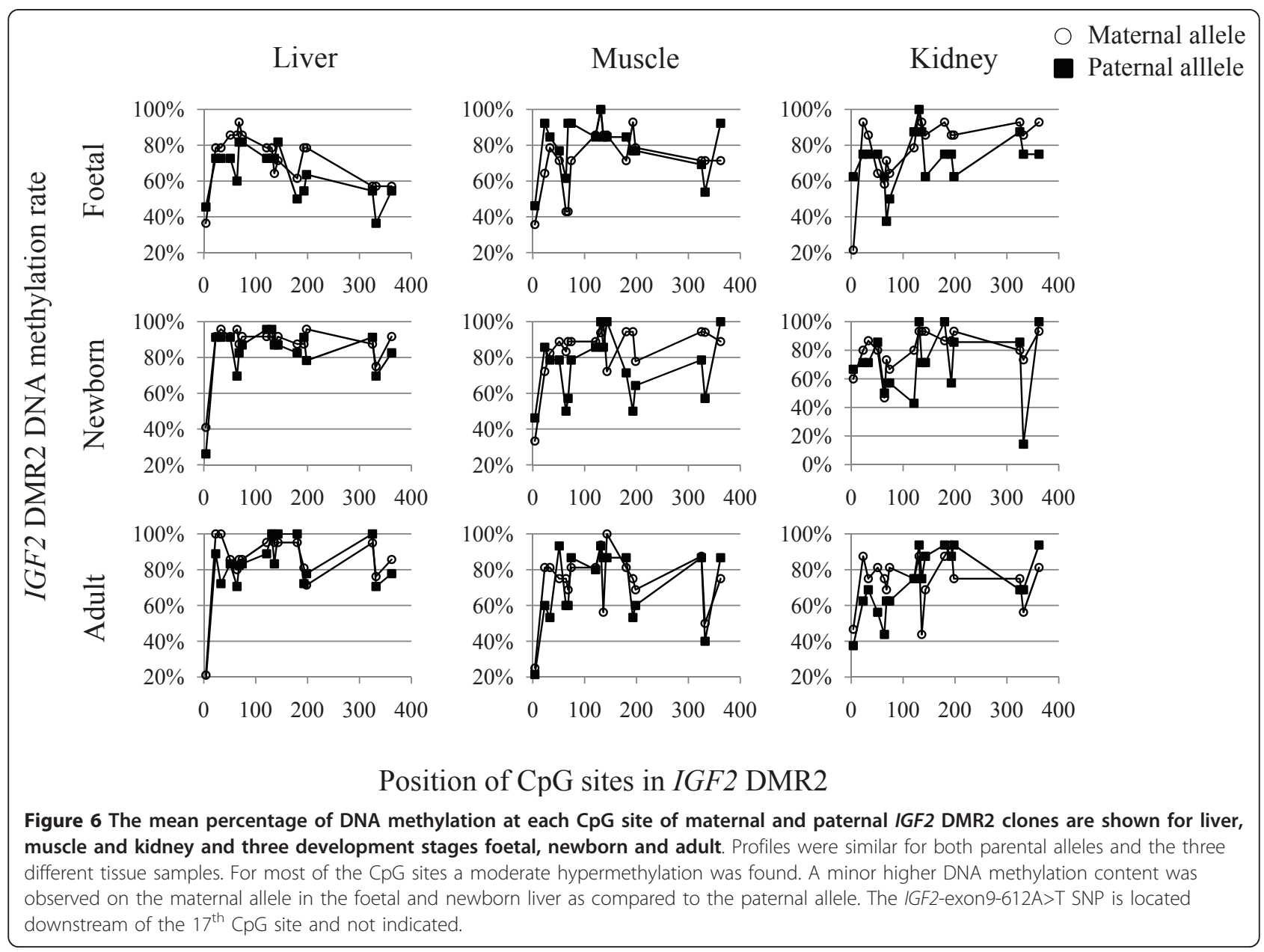


promoter P1 in heart, liver, brain, lung, kidney, stomach, pancreas, thymus, tongue, muscle, bladder, spleen, and placenta tissues of week-old pigs. Their RT-PCR analysis of microsatellite $S W C 9$ in these 13 different tissues revealed, however, exclusive or nearly exclusive paternal IGF2 expression. Furthermore, their real-time PCR analysis of IGF2 exon 2 originating from promoter P1 and of IGF2 exon 9, that is common for all IGF2 transcripts, resulted in roughly $33 \%$ and $10 \%$ promoter P1 IGF2 transcription relative to total IGF2 transcription in brain and placenta, respectively. These results are in agreement with the data presented for IGF2 imprinting in liver and suggest that transcription from the IGF2 promoter P1 may be regulated by other mechanisms than that from promoters P2, P3 and P4. Together these data emphasize the promoter-specific IGF2 imprinting status in different tissues and during development [16]. Biallelic expression was also observed in the liver and brain of 6-month-old lambs but not in their kidneys [22].

H19 DMD was paternally hypermethylated and maternally hypomethylated in liver, muscle and kidney of all three developmental stages independent of the combined imprinting status of all IGF2 expressed transcripts. This finding challenges the boundary model $[12,13]$ postulating that the vertebrate eleven-zinc finger protein CTCF binds the maternal unmethylated H19 DMD insulating the upstream IGF2 promoters from enhancers downstream of $H 19$. On the paternal allele the methylated DMD abolishes CTCF binding and enhancers 3' of $H 19$ have access to IGF2 promoters. Our results demonstrate that IGF2 is expressed from both alleles, mainly in adult liver and, to a much lesser extent in skeletal muscle and kidney, although H19 DMD is indeed differentially methylated. Histone modifications might still cause these effects but if so, they would be independent of DNA methylation. Investigations of mouse Igf2 DMR 1 and 2 led to a model of parent-specific chromatin loops that regulate Igf2 imprinting [14]. DNA methylation at DMR 1 and 2 in our samples does not support a parent-specific chromatin loop model regulating IGF2 imprinting in pig. It remains inconclusive if subtle differences in DNA methylation in DMR1 between foetal and adult liver and that between the parental alleles in DMR2 of foetal and newborn liver are involved in the control of the IGF2 imprinting status.

\section{Conclusions}

From our imprinting and DNA methylation analyses we conclude, firstly, that IGF2 expression from both parental alleles in adult porcine liver and relaxation of IGF2 imprinting in adult porcine muscle are not associated with DNA methylation variation at and around at least one CTCF binding site in H19 DMD. Secondly, the lower DNA methylation content in DMR1 in foetal liver, as compared to adult liver, should be evaluated on molecules from which the parental origin could be established. Thirdly, similar to DMR1 porcine DMR2 is hypermethylated on both parental alleles rather than differentially methylated, as observed for H19 DMD. Furthermore, the maternal DMR2 allele was more methylated in foetal and newborn animals when compared to the respective paternal allele. Finally, the transition of IGF2 imprinting in foetal liver to IGF2 expression from both alleles in adult liver may inherent new mechanisms involved in IGF2 imprinting regulation and provides a promising subject for further study.

\section{Methods}

\section{Animals}

From a collection of Swiss Large White pigs 2 male foetus, 1 female and 1 male newborn and 6 adult boars were selected based on their heterozygosity for the microsatellite marker SWC9 located in the 3'-UTR of the IGF2 gene.

\section{DNA and RNA isolation and first strand CDNA synthesis}

DNA was isolated from liver, skeletal muscle and kidney tissues using the DNeasy Blood \& Tissue Kit from Qiagen (Hombrechtikon, Switzerland). RNA extraction was performed with Trizol ${ }^{\circledR}$ Reagent according to the manufacturer's protocol (Invitrogen, Lucerne, Switzerland). Total RNA was digested with RNase-Free DNAse I according to the supplier's instructions (Ambion, Rotkreuz, Switzerland). DNA free RNA was reverse transcribed using a First-Strand cDNA Synthesis Kit (GE Healthcare, Glattbrug, Zurich) and products were subsequently purified with QIAquick columns (Qiagen).

\section{Imprinting status}

Imprinting status was investigated by means of the SWC9 microsatellite marker located in the 3'-UTR of the IGF2 gene (Figure 1). The SWC9 microsatellite marker was amplified using Qiagen's Multiplex PCR Master Mix including a FAM labeled forward primer. PCR was performed with an initial step at $94^{\circ} \mathrm{C}$ for 15 minutes and 34 cycles of a denaturation step at $94^{\circ} \mathrm{C}$ for $30 \mathrm{sec}-$ onds, an annealing step at $60^{\circ} \mathrm{C}$ for 1 and a half minutes and an elongation step at $72^{\circ} \mathrm{C}$ for 1 minute, and a final elongation for 10 minutes. All samples including the standard samples were analysed in triplicates and subjected to the same PCR run in a 96 well plate. Sequences of the PCR primers are shown in Supplementary Table 1 . Following PCR $1 \mu$ of each reaction was combined with $10 \mu \mathrm{l}$ of genotyping mix (980 $\mu \mathrm{l}$ of $\mathrm{HiDi}$ formamide and $20 \mu \mathrm{l}$ of GeneScanTM-500 LIZTM Size Standard (Applied Biosystems, Rotkreuz, Switzerland). The mixture was denatured for $2 \mathrm{~min}$., chilled on ice and loaded on an ABI 3730 capillary sequencer (Applied 
Biosystems). Data was analyzed using GeneMapper software version 4.0 (Applied Biosystems). A standard dilution series was established based on the peak areas of mixed DNA samples from two homozygous individuals for the respective SWC9 236 and SWC9 247 alleles. We used ratios of $32: 1,16: 1,8: 1,4: 1,2: 1,1: 1,1: 2,1: 4,1: 8$, $1: 16$, and $1: 32$ of the respective SWC9 236 and SWC9 247 homozygous DNA samples. The ratio of the peak areas was used to calculate the ratio between the SWC9 alleles 236 and 247 according to the formula $\mathrm{y}=$ $0.9519 x+0.014$ Figure 2 and[20].

\section{Bisulfite sequencing}

DNA was converted with the EpiTect Bisulfite kit according to the supplier's manual (Qiagen). Bisulfiteconversion-based methylation PCR primers were designed with the program Methprimer http://www.urogene.org/methprimer/index.html and in the case of IGF2_DMR2 with Methyl Primer Express (ABI). Primer sequences and product sizes of the 4 fragments covering the H19 DMD (H19_DMD_1, H19_DMD_2, H19_DMD_3, H19_DMD_4), the IGF2 DMR1 (IGF2_DMR1) and the IGF2 DMR2 (IGF2_DMR2) is shown in Additional file 1 Table S1. PCR was performed with the Multiplex PCR Master Mix and products from liver, skeletal muscle and kidney were cloned (TOPO TA Cloning Kit (Invitrogen). White colonies were picked diluted in $50 \mu \mathrm{l}$ water and amplified with the illustra $^{\mathrm{TM}}$ TempliPhi amplification kit (GE Healthcare) and sequenced on an ABI 3730 capillary sequencer (Applied Biosystems). Bisulfite sequencing analysis was performed with the programs BiQ Analyzer http://biqanalyzer.bioinf.mpi-sb.mpg.de/ and MethTools http:// genome.fli-leibniz.de/methtools/.

\section{DNA methylation analysis using methyl-sensitive \\ restriction endonuclease Sacll and 'hot-stop' PCR}

About 100 ng genomic DNA was digested over night with the methyl-sensitive SacII endonuclease (New England BioLabs, Allschwil, Switzerland). CpG methylation of the recognition site CCGCGG inhibits digestion. A SacII recognition site is present in a CTCF binding site 5' upstream of H19 and is referred to pig repeat P2 [5]. We previously re-sequenced the pig imprinting control region (ICR) containing the three pig CTCF binding sites upstream of H19. By this means we identified two SNPs, one at position AY044827.1:g.32530C $>$ T and the other at position AY044827.1:g.32619G>A. PCR primers ('hot-stop') were designed which encompass the CTCF binding site in P2 and the two SNPs (Supplemented Table 1). The forward primer is tailed with a M13 forward sequence. An aliquot of the SacII digested DNA was PCR amplified for each sample using these primers by a 'hot-stop' PCR procedure for linear quantification of allele ratios [23]. The 'hot-stop' PCR was performed with Multiplex PCR Master Mix (Qiagen) for 35 cycles with an initial denaturation step at $94^{\circ} \mathrm{C}$ for $15 \mathrm{~min}$ followed by denaturation at $94^{\circ} \mathrm{C}$ for $30 \mathrm{sec}$, an annealing temperature of $55^{\circ} \mathrm{C}$ for $30 \mathrm{sec}$, an annealing temperature of $68^{\circ} \mathrm{C}$ for $30 \mathrm{sec}$ and an elongation step at $72^{\circ} \mathrm{C}$ for $30 \mathrm{sec}$. The PCR was then paused after these 34 cycles at $72^{\circ} \mathrm{C}$ and $0.2 \mu \mathrm{M}$ IRDye ${ }^{\mathrm{TM}} 700$ labeled M13 primers was added to the reaction. The PCR was then resumed for an additional cycle and a final elongation step at $72^{\circ} \mathrm{C}$ for $10 \mathrm{~min}$.

The PCR product contains a common 5'...CGCG...3' recognition site for Bst $U \mathrm{I}$ (New England BioLabs) and a second that includes the AY044827.1:g.32530C > T SNP which was used to discriminate the parental origin of the alleles in this fragment of H19 DMD. An aliquot of the 'hot-stop' PCR was subsequently digested over night with BstUI according to the supplier's recommendation. The digested products were separated on a $1.5 \%$ agarose gel and scanned on an Odyssey Infrared Imaging System according to LI-COR's instruction (LI-COR Biosciences, Bad Homburg, Germany). Bands were visualized using the LI-COR Odyssey software.

\section{Parent of origin determination of alleles at the IGF2 DMR2}

To determine the allele origin of IGF2 DMR2 we used a single nucleotide polymorphism (SNPs) in the 3'-UTR at position IGF2-exon9-612A $>$ T. Based on this SNP we analyzed parental DNA methylation patterns at the IGF2 DMR2.

\section{Acknowledgements}

We thank Leeson J. Alexander for careful reading the manuscript and critical comments. This research project has been co-financed by the European Commission, within the 6th Framework Programme, contract no. FOOD-CT-2006-016 250. The text represents the authors' views and does not necessarily represent a position of the Commission, who will not be liable for the use made of such information.

\section{Additional material}

Additional file 1: Primer sequences, annealing temperatures and product sizes. The table contains PCR primer sequence information including annealing temperatures and product sizes.

\section{Authors' contributions}

MHB conceived and designed the study. MOL sequenced the clones and performed the expression study. NSS isolated DNA and RNA and did the bisulfite analysis. MHB performed the 'hot-start' PCR, the methylation analysis and wrote the manuscript. All authors read and approved the final manuscript. 
Received: 10 February 2011 Accepted: 17 May 2011

Published: 17 May 2011

\section{References}

1. DeChiara TM, Robertson EJ, Efstratiadis A: Parental imprinting of the mouse insulin-like growth factor II gene. Cell 1991, 64:849-859.

2. Bartolomei M, Zemel S, Tilghman SM: Parental imprinting of the mouse H19 gene. Nature 1991, 351:153-155.

3. Arney KL: H19 and Igf2-enhancing the confusion? Trends Genet 2003, 19:17-23.

4. DeChiara TM, Efstratiadis A, Robertson EJ: A growth-deficiency phenotype in heterozygous mice carrying an insulin-like growth factor II gene disrupted by targeting. Nature 1990, 345:78-80.

5. Amarger $V$, Nguyen M, Van Laere A-S, Braunschweig MC, Nezer C, Georges M, Andersson L: Comparative sequence analysis of the INS-IGF2H19 gene cluster in pigs. Mamm Genome 2002, 13:388-398.

6. Bartolomei MS, Vigneau S, O'Neill MJ: H19 in the pouch. Nat Gene 2008, 40:932-933.

7. Cai X, Cullen BR: The imprinted H19 noncoding RNA is a primary microRNA precursor. RNA 2007, 13:313-316.

8. Van Laere A-S, Nguyen M, Braunschweig M, Nezer C, Collette C, Moreau L, Archibald AL, Haley CS, Buys N, Tally M, Andersson G, Georges M, Andersson L: A regulatory mutation in IGF2 causes a major QTL effect on skeletal muscle growth in the pig. Nature 2003, 425:832-836.

9. Braunschweig MH, Van Laere A-S, Buys N, Andersson L, Andersson G: IGF2 antisense transcript expression in porcine postnatal skeletal muscle is affected by a quantitative trait nucleotide in intron 3. Genomics 2004, 84:1021-1029.

10. Tremblay KD, Saam JR, Ingram RS, Tilghman SM, Bartolomei MS: A paternal-specific methylation imprint marks the alleles of the mouse H19 gene. Nature Genet 1995, 9:407-413.

11. Thorvaldsen $J L$, Duran $\mathrm{KL}$, Bartolomei MS: Deletion of the $\mathrm{H} 19$ differentially methylated domain results in loss of imprinted expression of $\mathrm{H} 19$ and Igf2. Genes Dev 1998, 12:3693-3702.

12. Bell AC, Felsenfeld G: Methylation of a CTCF-dependent boundary controls imprinted expression of the lgf2 gene. Nature 2000, 405:482-485.

13. Hark AT, Schoenherr CJ, Katz DJ, Ingram RS, Levorse JM, Tilghman SM: CTCF mediates methylation-sensitive enhancer-blocking activity at the $\mathrm{H} 19$ / Igf2 locus. Nature 2000, 405:486-489.

14. Murrell A, Heeson S, Reik W: Interaction between differentially methylated regions partitions the imprinted genes Igf2 and $\mathrm{H} 19$ into parent-specific chromatin loops. Nat Genet 2004, 36:889-893.

15. Wrzeska M, Żyga A, Rejduch B, Słota E: A note on biallelic expression of the IGF2 gene in the liver and brain of adult pigs. J Anim Feed Sci 2006, 15:57-60.

16. Li C, Bin Y, Curchoe C, Yang L, Feng D, Jiang Q, O'Neill M, Tian XC, Zhang S: Genetic imprinting of H19 and IGF2 in domestic pigs (Sus scrofa). Anim Biotechnol 2008, 19:22-27.

17. Ekström TJ, Cui H, Li X, Ohlsson R: Promoter-specific IGF2 imprinting status and its plasticity during human liver development. Development 1995, 121:309-316

18. Li X, Cui H, Sandstedt B, Nordlinder H, Larsson E, Ekström TJ: Expression levels of the insulin-like growth factor-II gene (IGF2) in the human liver: developmental relationships of the four promoters. J Endocrinol 1996, 149:117-24.

19. Wu J, Qin Y, Li B, He WZ, Sun ZL: Hypomethylated and hypermethylated profiles of H19DMR are associated with the aberrant imprinting of IGF2 and H19 in human hepatocellular carcinoma. Genomics 2008, 91:443-450.

20. Lo HS, Wang Z, Hu Y, Yang HH, Gere S, Buetow KH, Lee MP: Allelic variation in gene expression is common in the human genome. Genome Res 2003, 13:1855-1862.

21. Sandovici I, Leppert M, Hawk PR, Suarez A, Linares Y, Sapienza C: Familial aggregation of abnormal methylation of parental alleles at the IGF2/H19 and IGF2R differentially methylated regions. Hum Mol Genet 2003, 12:1569-1578, Erratum in Hum Mol Genet 2004, 13:781.

22. McLaren RJ, Montgomery GW: Genomic imprinting of the insulin-like growth factor 2 gene in sheep. Mamm Genome 1999, 10:588-591.

23. Uejima H, Lee MP, Cui H, Feinberg AP: Hot-stop PCR: a simple and general assay for linear quantitation of allele ratios. Nat Genet 2000 25:375-376. do: 10.1186/1471-2156-12-47

Cite this article as: Braunschweig et al:: Relationship of porcine IGF2 imprinting status to DNA methylation at the H19 DMD and the IGF2 DMRs 1 and 2. BMC Genetics 2011 12:47.

\section{Submit your next manuscript to BioMed Central and take full advantage of:}

- Convenient online submission

- Thorough peer review

- No space constraints or color figure charges

- Immediate publication on acceptance

- Inclusion in PubMed, CAS, Scopus and Google Scholar

- Research which is freely available for redistribution 